2. Навигация с использованием ArUco-маркеров [Электронный ресурс]. Режим доступа: https://clever.copterexpress.com/ru/aruco.html

3. Вращение фигуры в 3-х мерном пространстве [Электронный ресурс]. Режим доступа: http://grafika.me/node/82

\title{
THE EXPERIENCE OF USING THE JOINT CONTROL DEVICES OF VOLTAGE AND REACTIVE POWER REGULATION ON A LABORATORY BENCH
}

\author{
Khmara G.A. ${ }^{*}$, Vlasova E.P. \\ Tyumen Industrial University, Tyumen, Russia \\ *E-mail: hmaraga@tyuiu.ru
}

The paper demonstrates an approach to the creation of the mathematical model of electric power transmission grid with using an artificial neural network for training a centralized system of ACS RPVC. The efficiency of its application was proved during the full-scale experiment on the laboratory bench.

Reactive Power and Voltage Control devices (RPVC) in power grids are used to reduce the loss of reactive power. There is a problem of increasing the reactive component of power while providing acceptable voltage values in the load nodes. The maintaining voltage in the load node at a given level leads to an increase in losses in power transmission grids, and the compensating devices used in them are intended for voltage regulation.

Today to solve this actual problem the degree of participation of devices regulating the RPVC is determined based on the criterion of optimization to minimize losses in the transmission of electricity. An alternative solution may be a joint management of devices regulating the RPVC, located in different nodes of the power grid to ensure minimal losses. JSC «NTC FGC UES» is developing decentralized multi-agent control system RPVC (MACS RPVC) where each agent is active and determines its contribution to the regulation.

The transition from centralized control systems to decentralized multi-agent automatic control systems (MACS) is associated with difficulties connected with insufficient study of the impact of each individual device of regulation of the RPVC on the power supply system and their interaction with each other in different modes. A transitional option between centralized and decentralized ACS RPVC should be power management systems. It is necessary to study the possibility of controlling the RPVC within a small power grid, creating active agents-dedicated areas of power supply systems. The allocation of active agents within the considered power grids will be the next step.

We show an approach to the creation of a mathematical model of the power grid using artificial neural network (ANN) for training of a centralized control system of ACS RPVC of a grid. To create a training sample of the neural network on the 
laboratory bench, a scheme of a power grid was designed. The structure of the power grid includes: the node of the power supply system (infinite power bus), taken as the basis; the load node, representing the active consumer with variable resistance; two nodes with connected synchronous generators, which in this case are reactive power control devices.

The results of this research showed the possibility of training ACS RPVC of a power grid with usage of ANN. Tests of the ACS RPVC allowed to prove the efficiency of the joint control devices regulating RPVC in the electrical power transmission.

\title{
CREATION OF A MEDICAL INFORMATION SEARCH SYSTEM IN THE ELECTRONIC MEDICAL CARD TO ASSIST IN DECISION-MAKING BY A DOCTOR
}

\author{
Khrushkov A.E. $^{{ }^{*}}{ }$, Ptuhin A.A. ${ }^{1}$ \\ 1) Ural Federal University, Yekaterinburg, Russia \\ *E-mail: deagle1@ mail.ru
}

Annotation. The purpose of this work is to create an assistant doctor, with which he can easily work with an electronic medical card, without spending too much time searching for the information he needs.

The doctor does not always see the whole picture, therefore, to decide or make the correct diagnosis, you need to visually look at the data that was collected during the examination of the patient. Be it a temperature chart, blood sugar or other tests.

There is a problem that could not be solved by introducing medical information systems. Such a problem is the analysis of the patient's card. With the introduction of an electronic medical record of the patient, the doctor must spend more time viewing the electronic medical record of the patient in search of the necessary tests and examinations than when using his paper equivalent. They tried to solve this problem by optimizing the interface, but this technique did not prove to be effective.

The healthcare system needs an informatization process. Informatization is a set of measures aimed at providing the participants of a given activity in a timely and complete manner with the necessary information, which has been processed in a certain way and, if necessary, transformed [1].

Informatization of the health care system is a multidimensional system-forming process, including the collection and accumulation of information, its transfer, integration, and the effective use of databases, databanks, and knowledge about the activities of the industry [2].

When writing an extension for Google Chrome, the main task was the choice of programming languages in which the front-end part of the application will be implemented. My choice fell on the following programming languages:

- JavaScript; 